\title{
IT applied to Public Lighting Management: How IT can improve public lighting management?
}

\author{
Nuno Seca ${ }^{1}$, Fernando Moreira ${ }^{1}$ \\ ${ }^{1}$ DICT, Universidade Portucalense, Porto, Portugal \\ nunoseca@schreder.pt,fmoreira@upt.pt
}

Keywords: Telemenagement, Efficient management of public lighting, PLC, ZigBee, Artificial Intelligence.

\begin{abstract}
Efficiency of public lighting management is getting relevant. Due to costs reduction "obligation" some entities have been falling in the temptation of power off street lighting. Once that this procedure puts in risk people and goods' security and considering that there are many lighting flux reduction solutions on the market it will be proposed a new approach. It was developed a prototype to allow managing of public lighting in an integrated way and based on Artificial Intelligence. Major goal is to achieve a higher level in which operational simplification can be made with significant cost reduction and operative optimization. Besides that, proposed approach, recurring to information systems' supported on neural networks and functional layers can be used on this particular domain contributing to benefit public lighting systems in a remarkable way concerning profitability return of investment and operationality.
\end{abstract}

\section{INTRODUCTION}

Public lighting is changing. Public lighting has always been based on traditional solutions supported on traditional lamps; however, lighting technology is going further.

Nowadays we are moving to LED technology and telemanagement solutions are gaining more and more relevance. Not so long ago, people's expectations were about having as much light as possible (Box, 2010). This feeling is changing and populations are getting concerned about energy consumption and the way to light better (Future, 2012).

We are finally assuming that good light is not strictly related with too much light. Environmental conscience is also getting relevance and lamps based on mercury are now assumed as an intense source of pollution and are becoming forbidden in several countries. Another aspect that is getting importance is the ability to requalify regular lighting installations with systems to vary lighting flux(assuming flux as the quantity of light emited by a lighting source in every directions) in accordance with several events such as time schedule or environment behaviors. Also important is that, when LED is used in public lighting, cost related with energy consumption can be reduced around $60 \%$ (and we have also to consider that TCO is much lower when compared with traditional lighting solutions) (Graves \& Ticleanu, 2011).

Even with LED technology (Lenk \& Lenk, 2011) and their biggest advantages, flux reduction can be made to achieve better result concerning energy cost reduction.

Based on previous considerations future of public lighting tends to be based on integrated solutions where protocol variation will be something regular and not a constraint. More than that and presuming that current "lighting intelligence" can go further than scheduled dimming scenarios or sensors able to make it react due to environment variation, we'll see (sooner or later) Artificial Intelligence assuming relevance on this business, and will assume relevance and will be revolutionary. Based on "learning from the past" public lighting will turn the page and a new chapter will be written.

As in many other areas IT is getting relevance. A few years ago, something like dynamic flux variation of public lighting would be interpreted like something that would never happen. However, nowadays, energy costs are becoming higher and higher and also due to global economic crisis, it's gaining significant relevance. On one hand, people are quite concern about ecological footprint and economical savings on the other hand, they don't want to lose their quality of life. We have to find a way to manage it and telemanagement and flux 
reduction are an option (and a reality) to achieve it. What is being proposed is to move forward and to make public lighting more intelligent, cheaper and easy to manage. This will be a reality. It's a question of time.

Due to all of that, this paper propose, it to go further and to present an advanced solution based on Artificial Intelligence and functional layers supported by a middleware platform able to manage and operate in a single way heterogeneous telemanagement lighting solutions based on PLC (CENELEC) and ZigBee (IEEE, 2011).

This paper is presented with the following approach: First of all, are presented relevant issues related technologies in use to achieve and implement telemanagement solutions. Then, we present a brief summary about ZigBee and PLC technologies. Following that it's proposed a solution to optimize the telemanagent and the way it can be done (based on heterogeneous technologies integration, Artificial Intelligence and functional layers). It ends with conclusion and future perspectives for public light intelligent management and the way to make it predictive instead of current approach based on reactivity.

\section{RELEVANT ISSUES}

Paradigms related with public lighting are changing faster and faster and the biggest players on the market are assuming positions to strike each other. Most of the biggest companies acting on lighting business are "dressing to impress" and trying to move as faster as they can into this new reality.

Some of them have already developed solutions based on technologies that are not new (except on lighting business). Some of them have chosen PLC (Sogexi), others made their option on ZigBee (Owlet) and many others are still figuring what is going to be the definitive move of the market to define their strategy. Future will reveal who made the best choice and most important than that, who will achieve better performance on sales and technology. However this is not the "one dollar question". From the economic and environmental point of view, citizens are hopping the best from lighting companies but we have to look further and put in question if lighting reduction based on predefined schedules is our major role and if it's the biggest goal to achieve.

Nowadays we have already solutions based on PLC or ZigBee to implement telemanagement to achieve flux reduction and, obviously, to reduce costs related with public lighting. However, what we need to have in mind is: Is this it?

\section{HOW IT GOES}

As said, paradigms related with public lighting are changing. Currently, if the goal is to implement telemanagement over public lighting to reduce costs related, we can find several solutions on the market.

Depending of the type of the luminaries, we can choose between Tension Reduction, PLC or ZigBee to achieve significant cost reduction. It's common sense that to reduce flux output we may reduce tension on the luminaries.

In a simple way, if we use $230 \mathrm{~V}$ to light a lamp it's expected to have an "amount" of light. Following this principle is reasonable that flux output will be reduced it tension used is also reduced (keep in mind that flux reduction and tension reduction does not perform as a linear function). This is probably the simplest way to achieve our goal: lighting cost reduction. It seems to be a good solution, isn't it? Seems to be simple, fast commissioning, and cheap. In fact, this is just like that and it works! But... it works well only if all the luminaries in each circuit are very similar between them.

An important aspect that cannot be jeopardized occurs when tension reduction is made. Some luminaries will reduce flux more than the others and it's almost certain that some luminaries will simply "switch off" due to tension reduction. (Note also that due to lamp technology tension reduction may impact on flickering and variation of lighting color. Due to this simple explanation, we have to assume that tension variation is an option to be taken in consideration but only when luminaries are quite similar).

Moving into another level of public lighting telemanagement, we have PLC and ZigBee. Both are supported by OSI model. PLC is a protocol to communicate over regular power lines. The network topology used is bus. This kind of communication is regulated by CENELEC and it has reduced frequencies available to be used. The PLC biggest advantage is that networks infrastructure (in majority of cases) already exists. However there are some disadvantages related with it: EMI (electromagnetic interference), bandwidth, noise and multipath.

Multipath and noise are quite important aspects to be taken in consideration once that power lines were not designed for data communication (M. 
Zimmermann, 2000). However it can be managed based on spread-spectrum techniques like DSSS (Direct Sequence), FHSS (Frequency Hopping), TH (Time Hopping) and MC (Multi-Carrier).

When we talk about ZigBee, we are talking about a completely different approach (Organization, 2008). Instead of wires we are on the wireless universe of capabilities (and taking advantage of this king of approach - note also that there are some disadvantages on that). In this case communication is based on radio frequency and mesh network is the topology in use. No matter which technology is used, it's possible to achieve the same: energy cost reduction based on dimming, in accordance with light flux output reduction. Architectural approach and ZigBee philosophy are quite different: Once that it uses RF (radio frequency) to implement communication it's based on a more sophisticated concept: instead of a bus network topology it uses a mesh network which is much more resilient and fault-tolerant. Communication speed is much higher (250 kbps) and aspects like noise or multipath are managed in an easy and efficient way.

\section{HOW IT COULD BE}

Once that telemanagement is already a reality and considering that Information Technology gave a significant contribution is it reasonable to assume that it's done. A shorter perspective would say that we did it but, in fact, we believe that we are quite far from what can be done. To have sensors to turn on lights when movement is detected, (or when sunset arrives or depending on many others environment behaviors) is not the future. It might look like but this is the present (Graves \& Ticleanu, 2011).Tests with it are already being made in some pilot plants.

The first step ahead will be done when real time integration of heterogeneous technologies occurs. So far, considering solutions based on PLC (Philipps, 2000); (T.C. Banwell, 2001) or ZigBee (Prasad, 1998); (Ata Elahi, 2009) the network topology stands on a master controller and "slaves" able to receive orders, make lighting reductions happen, and give feedback to the master. First problem to solve is how to integrate different masters (even when they share the same technology). Nowadays, two different luminaires, close to each other but depending on different masters may assume different behaviors just because masters are not able to communicate between them. The second problem to solve is how to integrate different technologies in a single telemanagement solution. It's quite understandable that any village or city (no matter how big they are) will not always adopt the same lighting telemanagement solution. It's not reasonable because a single technology is not always the best solution. So, assuming this, why should a responsible for the system access several platforms to do his job? What concerns us is not the amount of work. What concerns us is how to manage in an integrated way different systems and systems based on different technologies.

That's the reason why we have developed a prototype for a solution based on a middleware able to implement the necessary abstraction to allow these systems to be managed as one. More than that public lighting must be considered as something to be intelligent. Based on that presupposition we propose to manage it taking in consideration that luminaries can act as a neural network supported by functional layers able to influence these systems with a data such as:

- Traffic rules;

- Holidays' calendar;

- Shopping areas;

- Residential and campus;

- Waste collection;

- Many others aspects that might be particular and to related people living close to telemanagement lighting installations.

Based on this kind of approach, it's possible to have different kind of behaviors once that in spite of "intelligence" acquired by luminaries controlled by the system they will performance in accordance with environmental assumptions to allow the entire system much effective. Note that a lighting behavior in the middle of the night might be quite different when it happens in a school surrounding area, in a petrol station or in a residential area.

Table 1 shows relevant weaknesses and aspects that can be improved (beside parameters shown on table 1 it was also compared: communication technology, signal strength, frequency, signal modulation, weaknesses, resilience, communication speed, maximum length between nodes, network topology and ballast / driver protocol).

Table 1 - Weaknesses of PLC and ZigBee Lighting solutions

\begin{tabular}{|l|c|c|}
\hline & PLC & ZigBee \\
\hline $\begin{array}{l}\text { Unified control of luminaries? } \\
\text { (considering that exists more than } \\
\text { one master controller) }\end{array}$ & No & No \\
\hline Allows integration with protocols & No & No \\
\hline
\end{tabular}




\begin{tabular}{|l|l|l|}
\hline $\begin{array}{l}\text { used by other telemanagement } \\
\text { technologies? }\end{array}$ & & \\
\hline $\begin{array}{l}\text { Intelligent and adaptive reaction } \\
\text { to environmental changes? }\end{array}$ & No & No \\
\hline
\end{tabular}

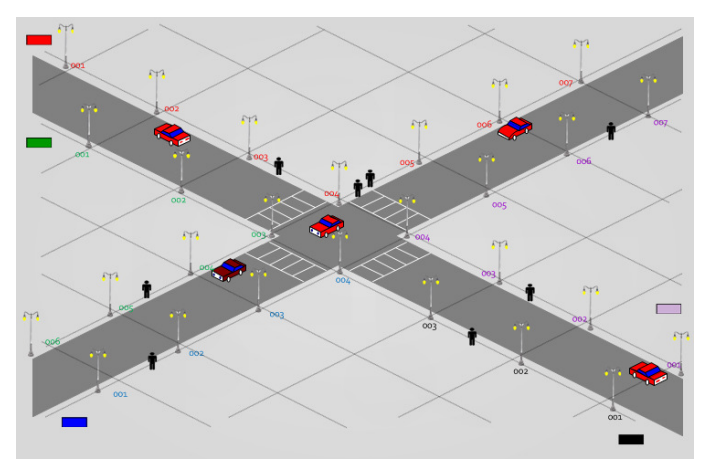

Figure 1 - Matrix approach for different technology integration

Figure 1 illustrates what is being proposed: Instead of different technological solutions that are blind to each other (illustrated with different colors), a logical matrix is created to allow interoperability for each luminaire. Independently of being managed by PLC or ZigBee, middleware takes in consideration what is the luminaire to be acted (for a sensor, for instance) and what luminaire is supposed to be acted based on how they are connected to each other and also based on function layers that supports Artificial Intelligence (Koch \& Segev, 2012) to operate the entire system. With this new approach, instead of different data speed of each systems, it's possible to manage it based on functional groups once that middleware only request to each master (no matter it works on Zigbee or PLC) to act a specific luminaire. Also note that with this approach and depending on how neural network (Norvig, 2003) is defined, luminaires can act as a group or individually. In this approach, neural network to be defined is quite important. Conceptually, it defines entrance nodes, nodes to be acted (middle neurons), and output nodes. (Decision to use neuronal networks is justified by the way they can handle advanced data analysis, by self-learning mechanisms, fault tolerance, capacity of generalization (even if some data is missing), ability to ignore "noise" data, adaptability and their major focus: to be used on real-time applications). Based on that, it's possible to define what luminaires must be acted when an entrance node is activated by behaviors detected that are supposed to be taken in consideration. Figure 2 illustrates the principle of neural networking.

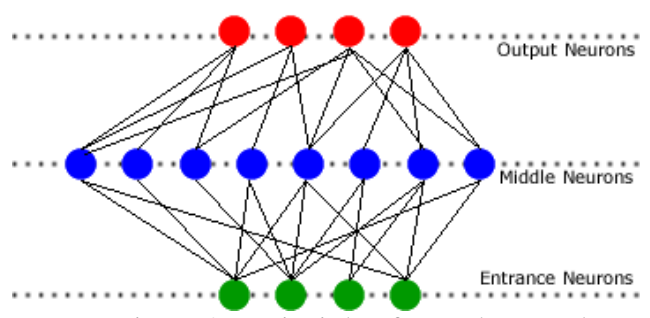

Figure 2 - Principle of neural network

As illustrated on figure 2, entrance neurons (sensors) are installed on main luminaries (typically luminaries installed in the beginning of the functional circuit and usually the first to be acted) that knows, based on relational information, what luminaries must be acted every time that a predetermined event occurs. Middle neurons are luminaries to acted by influence of a previous luminaire, and output neurons are luminaries (last in the circuit) that ends a predetermined path and closes the event that were triggered by the entrance neuron.

There is also another aspect to be considered: fading. Obviously, this kind of system must act in a smooth way. The way to achieve it is illustrated on figure 3 .

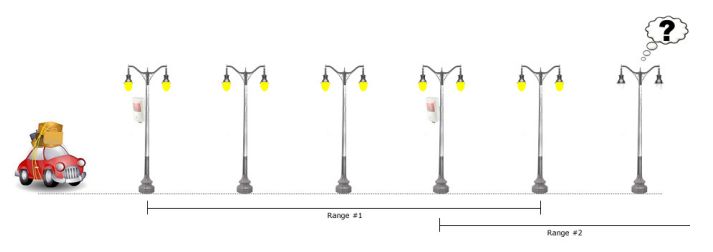

Figure 3 - Fading Effect Management

As illustrated on figure 3 , notice that there is an initial sensor (entrance neuron) that is informed of luminaries to be acted on a predefined range. The sensor to act the second group of luminaires is installed in a luminaire located into the first group range. When it detects behavior, the second group is activated before the arrival of the car.

As shown on figure 4, and considering that systems developed are installed separately (as they are already installed) and connected to their own "master system controller", development to be done is on the upstream of current systems. As shown, there is an "Integration Middleware" of different systems where described approach is implemented (Artificial Intelligence rules, functional and logical 


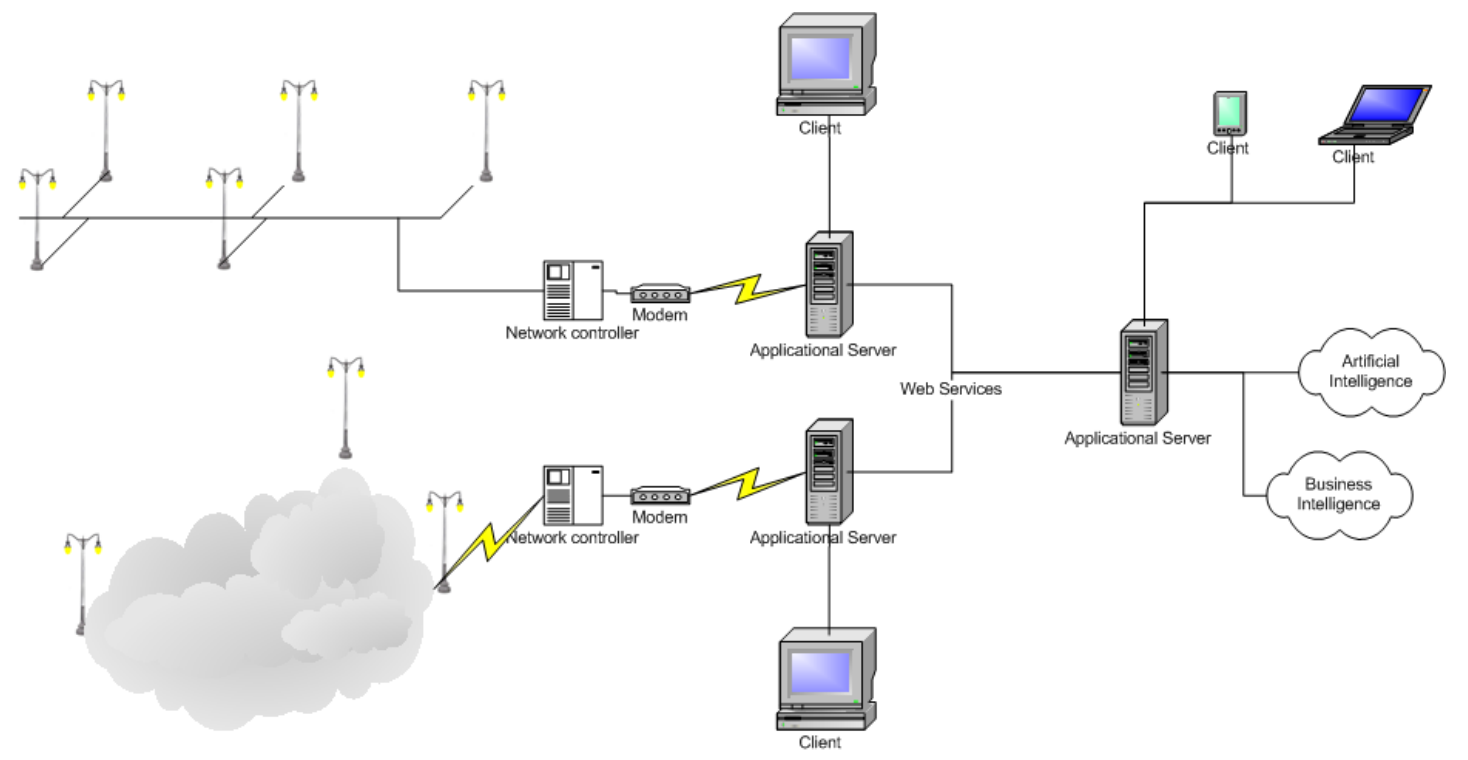

Figure 4 - Architectural Approach

layers, databases and the protocol translation). Besides that, and following what is already deployed by existing solutions, system will be able to be managed from local networks, Internet, VPN and by any type of devices (such as PC, laptops and mobile devices). The biggest goal, as said, is to provide a homogeneous platform able to provide single management that is independent from the "last mile" solution and able to manage public light in a predictive and non-static way.

When we think about the difference between sensors or schedule dimming scenarios when compared to AI it might be not immediate to distinguish between them but there is a big difference: AI makes it happens in a predictive way while sensors make it happen in a reactive way. Functionality is a reality on both systems but operability is completely different. There are many advantages if AI approach is taken in consideration. The biggest advantage, probably, is related with the ability to act in a predictive mode. In fact it forces the change of current paradigm. Telemanagement is being used as a brand new buzzword but in fact it's often confused with saving of energy consumption when, in fact, it only means that customer is able to reach (remotely) lighting systems and operate them.

On current telemanagement lighting systems, savings on energy costs are achieved based on flux reduction between certain periods of time. The goal is: if we have a $100 \%$ light output, let's reduce it during the hours when streets are (supposed to be) empty. A problem occurs when someone goes into the street in the middle of the night. Luminaries might be lighting to much low. The fastest answer to solve it is to install sensors able to detect movement and make luminaries react in accordance. In fact, it can be a good approach but it will be required to install as many sensors as many different behaviors can be taken in consideration. For instance: it will be required to install one sensor to detect if people move to left and another to detect if people move to the right. Once that current telemanagement lighting systems are reactive and "no-learning" it will never be able to predict movements and, based on that, it will never be possible to light only required luminaries and achieve a more effective cost reduction. Based on sensors there are no other approach than light a significant quantity of luminaries that are "enough" to answer to the more recurrent behaviours.

Resuming what was said, so far, and concerning LED technology it's possible to achieve significant saves on energy consumption. More than that, based on flux reduction schedule this value can be increased with significant impact also on total cost of ownership and ecological footprint. However, based on what is proposed public lighting performance can be optimized if some paradoxes and traditional approach is shifted. Based on what was already presented it's possible to change de drive from reactive lighting to predictive and more functional public lighting. 


\section{CONCLUSION AND FUTURE PERSPECTIVES}

Independently of what has been done and the evaluation made on technologies like PLC (Hrasnica, Haidine, \& Lehnert, 2004) or radio frequency like ZigBee (Walke, 1999), public lighting can go further and achieve higher levels of efficiency and usability. In the near future, people will be aware of it and will be focused on cost reduction, environmental footprint without jeopardizing security and public light performance.

Based on AI and principles of neural networks, (in this particular scenario and based on perception of previous behaviors, public lighting could always be reduced to minimum flux and pushed up every time that people needs light) instead of saving during part of the night, AI might implement an "always low" dimming profile and switch to a higher flux every time that light is really needed and light just only where light is required. Another very interesting advantage is that based on AI and neural networks, lighting groups can be dynamics instead of static.

There are numerous advantages like significant and effective cost reduction that will have impact on maintenance costs and energy consumption reduction. Other aspect quite important is that luminaries will last longer once that daily runtime hour might be reduced. Concerning environmental care there are many advantages once that $\mathrm{CO} 2$ emissions are reduced and luminaries will live longer and, obviously, won't need to be replaced so many times as they are used to.

More than that, the major question is about the maturity of customer to ask for this kind of solution and the capability and commercial interest of lighting players (notice that energy companies probably the most relevant players on lighting business - have significant profits based on energy selling and they make huge investments that are not intended to see underexploited) to provide it.

Proposed approach can be considered as a "nice to have" or something that will never become real but we have to remember that 5 years ago LED technology was not an option for public lighting. It has changed nowadays. It's common technology and the commercial differentiation is not significant because everyone has a "ready to use" solution. Lighting players' Differentiation will be done based on service. In fact, it's already been done. Currently, market drivers are changing and public lighting is becoming to be negotiated based on "lighting point performance" instead of quantity of supplied luminaires. Due to it, Public Lighting "intelligence" might be the most important argument once that it fits (and extends) most important aspects on public lighting: Return of Investment, profitability and operability.

\section{REFERENCES}

Ata Elahi, A. G. (2009). ZigBee Wireless Sensor and Control Network. Prentice Hall.

Box, H. C. (2010). Set Lighting Technician's Handbook. Focal Press.

Future, L. L. (2012). LED Lighting. O'Reilly Maker Press.

Graves, H., \& Ticleanu, C. (2011). LED Lighting: A Review of the Current Market and Future Developments . bre press.

Hrasnica, H., Haidine, A., \& Lehnert, R. (2004). Broadband Powerline Communications Networks. West Sussex, UK: John Wiley \& Sons, Lts.

IEEE. (2011). IEEE Standard for Local and metropolitan area networks. IEEE Computer Society.

Koch, C., \& Segev, I. (2012). Methods in Neuronal Modeling: From Ions to Networks. MIT Press.

Lenk, R., \& Lenk, C. (2011). Practical Lighting Design with LEDs. IEEE Press.

M. Zimmermann, K. D. (2000). The low voltage distribution network as last mile access network - sinal propagation and noise scenario in the HF range. AEÜ International Journal of Electronics and Communications.

Norvig, S. J. (2003). Artificial Intelligence. New Jersey: Prentice Hall.

Organization, Z. S. (2008). ZigBee Specification. ZigBee Standards Organization.

Owlet. (s.d.). Owlet. Retrived from http://owletstreetlight.de/en/.

Philipps, H. (2000). Development of a statistical model for powerline communications channels. Limerick, Ireleand: ISPLC.

Prasad, R. (1998). Universal Wireless Personal Communications. Boston, London: Artech House.

Sogexi. (s.d.). Sogexi. Retrived from http://www.sogexi.fr/.

T.C. Banwell, S. G. (2001). A new approach to the modelling of the transfer function of the power line chanel. Proceedings of the 5th International Symposium on Power-Line Communications and its Applications (ISPLC). Malmö. 